\title{
SPIN(9)-STRUCTURES AND CONNECTIONS WITH TOTALLY SKEW-SYMMETRIC TORSION
}

\author{
THOMAS FRIEDRICH
}

\begin{abstract}
We study Spin(9)-structures on 16-dimensional Riemannian manifolds and char-
\end{abstract} acterize the geometric types admitting a connection with totally skew-symmetric torsion.

\section{Contents}

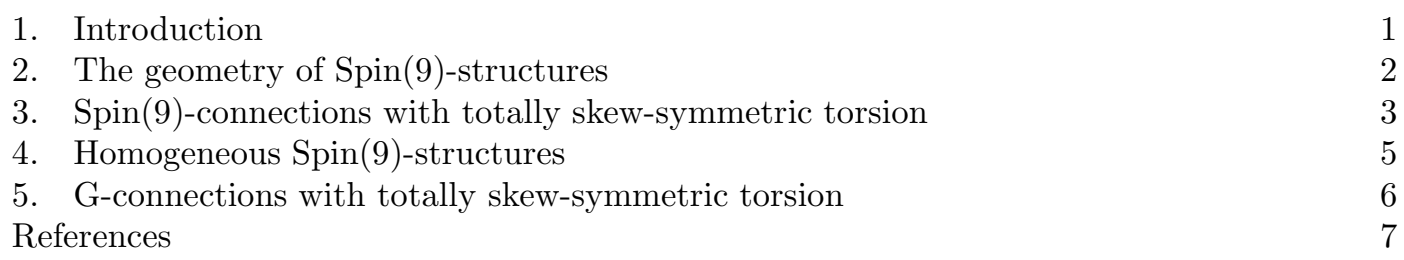

\section{INTRODUCTION}

The basic model in type II string theory is a 6 -tuple $\left(M^{n}, g, \nabla, T, \Phi, \Psi\right)$ consisting of a Riemannian metric $g$, a metric connection $\nabla$ with totally skew-symmetric torsion form $T$, a dilation function $\Phi$ and a spinor field $\Psi$. If the dilation function is constant, the string equations can be written in the following form (see [Stro] and [IP, FI1]):

$$
\operatorname{Ric}^{\nabla}=0, \quad \delta^{g}(T)=0, \quad \nabla \Psi=0, \quad T \cdot \Psi=0 .
$$

Therefore, an interesting problem is the investigation of metric connections with totally skewsymmetric torsion. In [FI1] we proved that several non-integrable geometric structures (almost contact metric structures, almost complex structures, $\mathrm{G}_{2}$-structures) admit a unique connection $\nabla$ preserving it with totally skew-symmetric torsion. Moreover, we computed the corresponding torsion form $T$ and we studied the integrability condition for $\nabla$-parallel spinors as well as the Ricci tensor Ric ${ }^{\nabla}$. In particular, we constructed 7-dimensional solutions of the string equations related to non-integrable $\mathrm{G}_{2}$-structures. The 5-dimensional case and its link with contact geometry was investigated in more details in the paper [FI2]. Similar results concerning 8-dimensional manifolds with a $\operatorname{Spin}(7)$-structure are contained in the paper [IV], the hyperkähler case was investigated in the papers DoFi], [IM] and Ver]. Homogeneous models and the relation to Kostant's cubic Dirac operators were discussed in [Agri]. The aim of this note is to work out the case of 16-dimensional Riemannian manifolds with a non-integrable Spin(9)-structure. Alfred Gray (see Gray]) has pointed out that this special geometry may occur as a geometry with a weak holonomy group. Only recently we once again revisited the special Spin(9)-geometries in dimension sixteen and, in particular, we proved that there are 4 basic classes (see [Fri1]). Here we will study the problem which of these classes admit a connection $\nabla$ with totally skew-symmetric torsion.

Received by the editors 22nd October 2018.

Key words and phrases. Spin(9)-structures, string equations.

Supported by the SFB 288 "Differential geometry and quantum physics" of the DFG. 


\section{The Geometry of Spin(9)-STRuctures}

The geometric types of Spin(9)-structures on 16-dimensional oriented Riemannian manifolds were investigated in the paper [Fri1]. We summerize the basic facts defining this special geometry. Let us consider the 16-dimensional oriented Euclidean space $\mathbb{R}^{16}$. This space is the real spin representation of the group $\operatorname{Spin}(9)$ and, therefore, there exist nine linear operators $I_{\alpha}: \mathbb{R}^{16} \rightarrow \mathbb{R}^{16}$ such that the following relations hold:

$$
I_{\alpha}^{2}=\mathrm{Id}, \quad I_{\alpha}^{*}=I_{\alpha}, \quad I_{\alpha} \cdot I_{\beta}+I_{\beta} \cdot I_{\alpha}=0 \quad(\alpha \neq \beta), \quad \operatorname{Tr}\left(I_{\alpha}\right)=0 .
$$

The subgroup $\operatorname{Spin}(9) \subset \mathrm{SO}(16)$ can be defined as the group of all automorphisms of $\mathbb{R}^{16}$ preserving, under conjugation, the 9 -dimensional subspace $\mathbb{R}^{9}:=\operatorname{Lin}\left\{I_{1}, \ldots, I_{9}\right\} \subset \operatorname{End}\left(\mathbb{R}^{16}\right)$,

$$
\operatorname{Spin}(9):=\left\{g \in \operatorname{SO}\left(\mathbb{R}^{16}\right): g \cdot \mathbb{R}^{9} \cdot g^{-1}=\mathbb{R}^{9}\right\} .
$$

The decomposition of the Lie algebra $\mathfrak{s o}(16)=\mathfrak{s o}(9) \oplus \mathfrak{m}$ is explicitly given by $\mathfrak{s o}(9):=\operatorname{Lin}\left\{I_{\alpha} \cdot I_{\beta}: \alpha<\beta\right\}=\Lambda^{2}\left(\mathbb{R}^{9}\right), \quad \mathfrak{m}:=\operatorname{Lin}\left\{I_{\alpha} \cdot I_{\beta} \cdot I_{\gamma}: \alpha<\beta<\gamma\right\}=\Lambda^{3}\left(\mathbb{R}^{9}\right)$.

The operators $I_{\alpha} \cdot I_{\beta}$ and $I_{\alpha} \cdot I_{\beta} \cdot I_{\gamma}$ are skew-symmetric and, consequently, they define two systems of 2-forms $\omega_{\alpha \beta}$ and $\sigma_{\alpha \beta \gamma}$.

Let $\left(M^{16}, g\right)$ be an oriented, 16-dimensional Riemannian manifold. A Spin(9)-structure is a 9-dimensional subbundle $V^{9} \subset \operatorname{End}\left(T M^{16}\right)$ of endomorphisms which is locally generated by sections $I_{\alpha}$ satisfying the algebraic relations described before. Denote by $\mathcal{F}\left(M^{16}\right)$ the frame bundle of the oriented Riemannian manifold. Equivalently, a Spin(9)-structure is a reduction $\mathcal{R} \subset \mathcal{F}\left(M^{16}\right)$ of the principal fibre bundle to the subgroup $\operatorname{Spin}(9)$. The Levi-Civita connection is a 1 -form on $\mathcal{F}\left(M^{16}\right)$ with values in the Lie algebra $\mathfrak{s o}(16)$,

$$
Z: T\left(\mathcal{F}\left(M^{16}\right)\right) \longrightarrow \mathfrak{s o}(16) .
$$

We restrict the Levi-Civita connection to a fixed $\operatorname{Spin}(9)$-structure $\mathcal{R}$ and decompose it with respect to the decomposition of the Lie algebra $\mathfrak{s o}(16)$ :

$$
\left.Z\right|_{T(\mathcal{R})}:=Z^{*} \oplus \Gamma
$$

Then, $Z^{*}$ is a connection in the principal $\operatorname{Spin}(9)$-bundle $\mathcal{R}$ and $\Gamma$ is a tensorial 1 -form of type Ad, i. e., a 1 -form on $M^{16}$ with values in the associated bundle

$$
\mathcal{R} \times_{\operatorname{Spin}(9)} \mathfrak{m}=\mathcal{R} \times_{\operatorname{Spin}(9)} \Lambda^{3}\left(\mathbb{R}^{9}\right)=\Lambda^{3}\left(V^{9}\right) .
$$

The Spin(9)-representation $\mathbb{R}^{16} \otimes \mathfrak{m}=\mathbb{R}^{16} \otimes \Lambda^{3}\left(\mathbb{R}^{9}\right)$ splits into four irreducible components,

$$
\mathbb{R}^{16} \otimes \mathfrak{m}=\mathbb{R}^{16} \oplus \mathcal{P}_{1}\left(\mathbb{R}^{9}\right) \oplus \mathcal{P}_{2}\left(\mathbb{R}^{9}\right) \oplus \mathcal{P}_{3}\left(\mathbb{R}^{9}\right)
$$

and, therefore, we obtain a similar decomposition of the bundle $\Lambda^{1}\left(M^{16}\right) \otimes \Lambda^{3}\left(V^{9}\right)$. The representation $\mathcal{P}_{1}\left(\mathbb{R}^{9}\right)$ has dimension 128 . It is the restriction of the half spin representation $\Delta_{16}^{-}$ of $\operatorname{Spin}(16)$ to the subgroup $\operatorname{Spin}(9)$. The dimensions of the irreducible representations $\mathcal{P}_{2}\left(\mathbb{R}^{9}\right)$ and $\mathcal{P}_{3}\left(\mathbb{R}^{9}\right)$ are 432 and 768 , respectively.

The decomposition of the section $\Gamma$ yields the classification of all geometric types of $\operatorname{Spin}(9)$ structures. In particular, there are four basic classes (see [Fri]). We remark that the sum $\mathcal{P}_{1} \oplus \mathcal{P}_{2}$ is isomorphic to the bundle of 3 -forms on $M^{16}$,

$$
\Lambda^{3}\left(M^{16}\right)=\mathcal{P}_{1}\left(V^{9}\right) \oplus \mathcal{P}_{2}\left(V^{9}\right)
$$

In order to fix the normalization, let us describe the embeddings $\Lambda^{i}\left(M^{16}\right) \longrightarrow \Lambda^{1}\left(M^{16}\right) \otimes$ $\Lambda^{3}\left(V^{9}\right), i=1,3$, by explicit formulas. If $\mu^{1} \in \Lambda^{1}\left(M^{16}\right)$ is a (co-)vector, then the 1 -form on $M^{16}$ with values in the bundle $\Lambda^{3}\left(V^{9}\right)$ is given by

$$
\mu^{1} \longmapsto \frac{1}{8} \sum_{\alpha<\beta<\gamma}^{9} I_{\alpha} I_{\beta} I_{\gamma}\left(\mu^{1}\right) \otimes I_{\alpha} \cdot I_{\beta} \cdot I_{\gamma} .
$$


Similarly, if $\mu^{3} \in \Lambda^{3}\left(M^{16}\right)$ is a 3 -form, we define

$$
\left.\mu^{3} \longmapsto \frac{1}{8} \sum_{\alpha<\beta<\gamma}^{9}\left(\sigma_{\alpha \beta \gamma}\right\lrcorner \mu^{3}\right) \otimes I_{\alpha} \cdot I_{\beta} \cdot I_{\gamma},
$$

where $\left.\sigma_{\alpha \beta \gamma}\right\lrcorner \mu^{3}$ denotes the inner product of the 2-forms $\sigma_{\alpha \beta \gamma}$ by $\mu^{3}$.

\section{SPIn(9)-CONNECTIONS With TOTALLY SKEW-SYMMETRIC TORSION}

We introduce the following equivariant maps:

$$
\begin{array}{ccl}
\Phi: & \mathbb{R}^{16} \otimes \mathfrak{s p i n}(9) \rightarrow \mathbb{R}^{16} \otimes S^{2}\left(\mathbb{R}^{16}\right), & \Phi(\Sigma)(X, Y, Z):=g(\Sigma(Z)(X), Y)+g(\Sigma(Y)(X), Z), \\
\Psi: & \mathbb{R}^{16} \otimes \mathfrak{m} \rightarrow \mathbb{R}^{16} \otimes S^{2}\left(\mathbb{R}^{16}\right), & \Psi(\Gamma)(X, Y, Z):=g(\Gamma(Y)(X), Z)+g(\Gamma(Z)(X), Y) .
\end{array}
$$

It is well known (see FI1]) that a geometric $\operatorname{Spin}(9)$-structure admits a connection $\nabla$ with totally skew-symmetric torsion if and only if $\Psi(\Gamma)$ is contained in the image of the homomorphism $\Phi$. The representation $\mathbb{R}^{16} \otimes \mathfrak{s p i n}(9)$ splits into

$$
\mathbb{R}^{16} \otimes \mathfrak{s p i n}(9)=\mathbb{R}^{16} \oplus \mathcal{P}_{1}\left(\mathbb{R}^{9}\right) \oplus \mathcal{P}_{2}\left(\mathbb{R}^{9}\right) .
$$

Consequently, if a $\operatorname{Spin}(9)$-structure admits a connection $\nabla$ with totally skew-symmetric torsion, then the $\mathcal{P}_{3}$-part of the form $\Gamma$ must vanish. We split the $\operatorname{Spin}(9)$-representation $\mathbb{R}^{16} \otimes S^{2}\left(\mathbb{R}^{16}\right)$ into irreducible components. Since the symmetric linear maps $I_{\alpha}$ are traceless, the representation $\mathbb{R}^{9}$ is contained in $S_{0}^{2}\left(\mathbb{R}^{16}\right)$ and we obtain the decomposition (see [Fri]])

$$
\mathbb{R}^{16} \otimes S^{2}\left(\mathbb{R}^{16}\right)=\mathbb{R}^{16} \oplus \mathbb{R}^{16} \otimes\left(\mathbb{R}^{9} \oplus D^{126}\right)=2 \cdot \mathbb{R}^{16} \oplus \mathcal{P}_{1}\left(\mathbb{R}^{9}\right) \oplus \mathbb{R}^{16} \otimes D^{126}
$$

where $D^{126}:=\Lambda^{4}\left(\mathbb{R}^{9}\right)$ is the unique irreducible representation of $\operatorname{Spin}(9)$ in dimension 126 . Denote by $D^{672}$ the unique irreducible Spin(9)-representation of dimension 672 . Its highest weight is the 4 -tuple $(3 / 2,3 / 2,3 / 2,3 / 2)$.

Lemma 3.1. The $\operatorname{Spin}(9)$-representation $\mathbb{R}^{16} \otimes S^{2}\left(\mathbb{R}^{16}\right)$ splits into the irreducible components

$$
\mathbb{R}^{16} \otimes S^{2}\left(\mathbb{R}^{16}\right)=3 \cdot \mathbb{R}^{16} \oplus 2 \cdot \mathcal{P}_{1}\left(\mathbb{R}^{9}\right) \oplus \mathcal{P}_{2}\left(\mathbb{R}^{9}\right) \oplus \mathcal{P}_{3}\left(\mathbb{R}^{9}\right) \oplus D^{672} .
$$

Proof. Since $\mathbb{R}^{16} \otimes \mathfrak{m}$ contains the representations $\mathcal{P}_{2}\left(\mathbb{R}^{9}\right), \mathcal{P}_{3}\left(\mathbb{R}^{9}\right)$ and $\Psi$ is nontrivial, the tensor product $\mathbb{R}^{16} \otimes D^{126}$ contains the two representations, too. Moreover, the highest weights of $\mathbb{R}^{16}$ and $D^{126}$ are $(1 / 2,1 / 2,1 / 2,1 / 2)$ and $(1,1,1,1)$, respectively. Then the tensor product $\mathbb{R}^{16} \otimes D^{126}$ contains the representation $D^{672}$ of highest weight $(3 / 2,3 / 2,3 / 2,3 / 2)$ (see [FH], page 425). Consequently, we obtain

$$
\mathbb{R}^{16} \otimes D^{126}=\mathcal{P}_{2}\left(\mathbb{R}^{9}\right) \oplus \mathcal{P}_{3}\left(\mathbb{R}^{9}\right) \oplus D^{672} \oplus S,
$$

where the dimension of the rest equals $\operatorname{dim}(S)=144$. The representation $S$ is not an $\operatorname{SO}(9)$ representation. The list of small-dimensional $\operatorname{Spin}(9)$-representations yields that $S=\mathbb{R}^{16} \oplus$ $\mathcal{P}_{1}\left(\mathbb{R}^{9}\right)$, the final result. The decomposition of $\mathbb{R}^{16} \otimes D^{126}$ can be computed by a suitable computer program, too.

Lemma 3.2. For any two vectors $X, Y \in \mathbb{R}^{16}$ the following identity holds:

$$
\sum_{\alpha<\beta}^{9} \omega_{\alpha \beta}(X, Y) \cdot \omega_{\alpha \beta}+\sum_{\alpha<\beta<\gamma}^{9} \sigma_{\alpha \beta \gamma}(X, Y) \cdot \sigma_{\alpha \beta \gamma}=8 \cdot X \wedge Y .
$$

Proof. The 2-forms $\omega_{\alpha \beta}$ and $\sigma_{\alpha \beta \gamma}$ constitute a basis of the space $\Lambda^{2}\left(\mathbb{R}^{16}\right)$ of all 2-forms in sixteen variables. Therefore, the identity is simply the decomposition of the 2 -form $X \wedge Y$ with respect to this basis. Remark that the length of the basic forms $\omega_{\alpha \beta}$ and $\sigma_{\alpha \beta \gamma}$ equals $2 \cdot \sqrt{2}$.

Theorem 3.1. A Spin(9)-structure on a 16-dimensional Riemannian manifold $M^{16}$ admits a connection $\nabla$ with totally skew-symmetric torsion if and only if the $\left(\mathbb{R}^{16} \oplus \mathcal{P}_{3}\right)$-part of the form $\Gamma$ vanishes. In this case $\Gamma$ is a usual 3 -form on the manifold $M^{16}$, the connection $\nabla$ is unique and its torsion form $T$ is given by the formula $T=-2 \cdot \Gamma$. 
Proof. For a fixed vector $\Gamma \in \mathbb{R}^{16}$ the tensor $\Psi(\Gamma)(X, Y, Y)$ is given by the formula

$$
\Psi(\Gamma)(X, Y, Y)=\frac{1}{4} \sum_{\alpha<\beta<\gamma}^{9} \sigma_{\alpha \beta \gamma}(\Gamma, Y) \cdot \sigma_{\alpha \beta \gamma}(X, Y) .
$$

Since the multiplicity of $\mathbb{R}^{16}$ in the representation $\mathbb{R}^{16} \otimes \mathfrak{s p i n}(9)$ equals one, any $\operatorname{Spin}(9)$ equivariant map $\Sigma: \mathbb{R}^{16} \rightarrow \mathbb{R}^{16} \otimes \mathfrak{s p i n}(9)$ is a multiple of

$$
\Sigma(\Gamma)=\sum_{\alpha<\beta}^{9} I_{\alpha \beta}(\Gamma) \otimes I_{\alpha \beta} .
$$

Consequently, if $\Psi(\Gamma)$ is in the image of $\Phi$, there exists a constant $c$ such that

$$
\sum_{\alpha<\beta<\gamma}^{9} \sigma_{\alpha \beta \gamma}(\Gamma, Y) \cdot \sigma_{\alpha \beta \gamma}(X, Y)=c \cdot \sum_{\alpha<\beta}^{9} \omega_{\alpha \beta}(\Gamma, Y) \cdot \omega_{\alpha \beta}(X, Y) .
$$

For $\Gamma=X=e_{16}$ we compute the corresponding quadratic forms in the variables $y_{1}, \ldots, y_{16}$ :

$$
\Psi\left(e_{16}\right)=\sum_{i=1}^{8} y_{i}^{2}+4 \cdot \sum_{j=9}^{15} y_{j}^{2}, \quad \Phi\left(\Sigma\left(e_{16}\right)\right)=7 \cdot \sum_{i=1}^{8} y_{i}^{2}+4 \cdot \sum_{j=9}^{15} y_{j}^{2},
$$

a contradiction. Next consider the case that $\Gamma \in \Lambda^{3}\left(\mathbb{R}^{16}\right)$ is a 3 -form. By Lemma 3.2 we have $\Psi(\Gamma)(X, Y, Y)=\frac{1}{4} \sum_{\alpha \beta \gamma}^{9} \Gamma\left(\sigma_{\alpha \beta \gamma}, Y\right) \cdot \sigma_{\alpha \beta \gamma}(X, Y)=-\frac{1}{4} \sum_{\alpha \beta}^{9} \Gamma\left(\omega_{\alpha \beta}, Y\right) \cdot \omega_{\alpha \beta}(X, Y)+2 \cdot \Gamma(X, Y, Y)$.

Since $\Gamma$ is a 3 -form, the term $\Gamma(X, Y, Y)$ vanishes. Let us introduce

$$
\left.\Sigma(\Gamma):=-\frac{1}{8} \sum_{\alpha \beta}^{9}\left(\omega_{\alpha \beta}\right\lrcorner \Gamma\right) \otimes \omega_{\alpha \beta} .
$$

Then $\Sigma(\Gamma)$ belongs to the space $\mathbb{R}^{16} \otimes \mathfrak{s p i n}(9)$ and we have $\Phi(\Sigma(\Gamma))=\Psi(\Gamma)$. Consequently, in case $\Gamma$ is a 3 -form on $M^{16}$, there exists a unique connection $\nabla$ preserving the $\operatorname{Spin}(9)$-structure with totally skew-symmetric torsion. Its torsion form $T$ is basically given by the difference $\Gamma(X)-\Sigma(\Gamma)(X)$ (see [FI1]) and we obtain the formula $T=-2 \cdot \Gamma$.

Let us characterize $\operatorname{Spin}(9)$-structures of type $\mathcal{P}_{1} \oplus \mathcal{P}_{2}$ using the Riemannian covariant derivatives $\nabla I_{\alpha}$ of the symmetric endomorphisms describing the structure. For an arbitrary 2-form $S$ we introduce the symmetric forms by the formula

$$
S_{\alpha}(Y, Z):=-S\left(I_{\alpha}(Y), Z\right)+S\left(Y, I_{\alpha}(Z)\right), \quad \alpha=1, \ldots 9 .
$$

The connection $\nabla$ preserves the 9-dimensional bundle of endomorphisms $I_{\alpha}$ and therefore there exist 1-forms $M_{\alpha \beta}$ such that $\nabla I_{\alpha}=\sum_{\beta=1}^{9} M_{\alpha \beta} \cdot I_{\beta}$. Since $\nabla_{X} Y=\nabla_{X}^{g}+\frac{1}{2} \cdot T(X, Y,$.$) we$ obtain the following formula for the Riemannian covariant derivative of the endomorphisms $I_{\alpha}$

$$
\left.\nabla_{X}^{g} I_{\alpha}=\sum_{\beta=1}^{9} M_{\alpha \beta}(X) \cdot I_{\beta}+\frac{1}{2} \cdot(X\lrcorner T\right)_{\alpha}
$$

where $T$ is a 3 -form. The latter equation characterizes $\operatorname{Spin}(9)$-structures of type $\mathcal{P}_{1} \oplus \mathcal{P}_{2}$. 


\section{Homogeneous Spin(9)-Structures}

Consider a Lie group $G$, a subgroup $H$ and suppose that the homogeneous space $G / H$ is naturally reductive of dimension 16 . We fix a decomposition

$$
\mathfrak{g}=\mathfrak{h} \oplus \mathfrak{n}, \quad[\mathfrak{h}, \mathfrak{n}] \subset \mathfrak{n}, \quad \mathfrak{n}=\mathbb{R}^{16}
$$

as well as a scalar product $(,)_{\mathfrak{n}}$ such that for all $X, Y, Z \in \mathfrak{n}$

$$
\left([X, Y]_{\mathfrak{n}}, Z\right)_{\mathfrak{n}}+\left(Y,[X, Z]_{\mathfrak{n}}\right)_{\mathfrak{n}}=0
$$

holds, where $[X, Y]_{\mathfrak{n}}$ denotes the $\mathfrak{n}$-part of the commutator. Moreover, suppose that the isotropy representation leaves a $\operatorname{Spin}(9)$-structure in the vector space $\mathfrak{n}$ invariant. Then $G / H$ admits a homogeneous Spin(9)-structure. Indeed, the frame bundle is an associated bundle,

$$
\mathcal{F}(G / H)=G \times{ }_{\mathrm{Ad}} \mathrm{SO}(\mathfrak{n}),
$$

and $\mathcal{R}:=G \rightarrow \mathcal{F}(G / H)$ is a reduction to the subgroup $H$ contained in $\operatorname{Spin}(9)$. The canonical connection $\nabla^{\text {can }}$ of the reductive space preserves the Spin(9)-structure and has totally skewsymmetric torsion,

$$
T^{\nabla^{c a n}}(X, Y, Z)=-\left([X, Y]_{\mathfrak{n}}, Z\right)_{\mathfrak{n}} .
$$

Consequently, any homogeneous Spin(9)-structure admits an affine connection with totally skew-symmetric torsion, i. e., it is of type $\mathcal{P}_{1} \oplus \mathcal{P}_{2}$.

Corollary 4.1. Any homogeneous $\operatorname{Spin}(9)$-structure on a naturally reductive space $M^{16}=G / H$ is of type $\mathcal{P}_{1} \oplus \mathcal{P}_{2}$.

Remark 4.1. In particular, for any homogeneous $\operatorname{Spin}(9)$-structure the difference $\Gamma$ between the Levi-Civita connection and the canonical connection is a 3 -form. Indeed, the Levi-Civita connection of a reductive space is given by the map $\mathfrak{n} \rightarrow \operatorname{End}(\mathfrak{n})$

$$
X \longmapsto \frac{1}{2} \cdot[X, \cdot]_{\mathfrak{n}} .
$$

Then we obtain

$$
\Gamma(X)=\frac{1}{2} \cdot \operatorname{pr}_{\mathfrak{m}}\left([X, \cdot]_{\mathfrak{n}}\right)=\frac{1}{32} \sum_{i, j=1}^{16} \sum_{\alpha<\beta<\gamma}\left(\left[X, e_{i}\right]_{\mathfrak{n}}, e_{j}\right)_{\mathfrak{n}} \cdot \sigma_{\alpha \beta \gamma}\left(e_{i}, e_{j}\right) \cdot \sigma_{\alpha \beta \gamma} .
$$

We write the latter equation in the following form

$$
\left.\Gamma(X)=-\frac{1}{16} \sum_{\alpha<\beta<\gamma}\left(\sigma_{\alpha \beta \gamma}\right\lrcorner T^{\nabla^{c a n}}\right)(X) \cdot \sigma_{\alpha \beta \gamma}=-\frac{1}{2} \cdot T^{\nabla^{c a n}}(X, \cdot, \cdot),
$$

i. e., $\Gamma$ is proportional to the torsion of the canonical connection,

$$
\Gamma(X)(Y, Z)=-\frac{1}{2} \cdot T^{\nabla^{c a n}}(X, Y, Z) .
$$

There are homogeneous Spin(9)-structures on different reductive spaces (see [Fri]).

Example 4.1. The group $\operatorname{Spin}(9)$ acts transitively on the sphere $S^{15}$, the isotropy group is isomorphic to $\operatorname{Spin}(7)$ and the isotropic representation of the reductive space $S^{1} \times S^{15}=$ $\left(S^{1} \times \operatorname{Spin}(9)\right) / \operatorname{Spin}(7)$ is contained in $\operatorname{Spin}(9)$.

Example 4.2. The space $S^{1} \times S^{1} \times\left(\mathrm{SO}(8) / \mathrm{G}_{2}\right)$ admits a homogeneous $\operatorname{Spin}(9)$-structure.

Example 4.3. The space SU(5)/SU(3) admits a homogeneous Spin(9)-structure. 


\section{G-CONNECTIONS WITH TOTALLY SKEW-SYMMETRIC TORSION}

The class of Spin(9)-structures corresponding to the representation $\mathbb{R}^{16} \subset \mathbb{R}^{16} \otimes \mathfrak{m}$ is related with conformal changes of the metric. Indeed, if $\left(M^{16}, g, V^{9}\right)$ is a Riemannian manifold with a fixed $\operatorname{Spin}(9)$-structure $V^{9} \subset \operatorname{End}\left(T M^{16}\right)$ and $g^{*}=e^{2 f} \cdot g$ is a conformal change of the metric, then the triple $\left(M^{16}, g^{*}, V^{9}\right)$ is a Riemannian manifold with a $\operatorname{Spin}(9)$-structure, too. The fact that the 16-dimensional class of $\operatorname{Spin}(9)$-structures corresponding to $\mathbb{R}^{16}$ is not admissible in Theorem 3.1 means that the existence of a connection with totally skew-symmetric torsion and preserving a Spin(9)-structure is not invariant under conformal transformations of the metric. From this point of view the behavior of $\operatorname{Spin}(9)$-structures is different from the behavior of $\mathrm{G}_{2^{-}}$ structures, Spin(7)-structures, quaternionic Kähler structures or contact structures (see [FI2], [FI3], [Iv], IM]). We will explain this effect in a more general context.

Let $\mathrm{G} \subset \mathrm{SO}(n)$ be a closed subgroup of the orthogonal group and decompose the Lie algebra

$$
\mathfrak{s o}(n)=\mathfrak{g} \oplus \mathfrak{m}
$$

A G-structure of a Riemannian manifolds $M^{n}$ is a reduction $\mathcal{R} \subset \mathcal{F}\left(M^{n}\right)$ of the frame bundle to the subgroup G. The Levi-Civita connection is a 1-form $Z$ on $\mathcal{F}\left(M^{n}\right)$ with values in the Lie algebra $\mathfrak{s o}(n)$. We restrict the Levi-Civita connection to a fixed G-structure $\mathcal{R}$ and decompose it with respect to the decomposition of the Lie algebra $\mathfrak{s o}(n)$ :

$$
\left.Z\right|_{T(\mathcal{R})}:=Z^{*} \oplus \Gamma \text {. }
$$

Then, $Z^{*}$ is a connection in the principal G-bundle $\mathcal{R}$ and $\Gamma$ is a tensorial 1-form of type Ad, i. e., a 1-form on $M^{n}$ with values in the associated bundle $\mathcal{R} \times{ }_{\mathrm{G}} \mathfrak{m}$. The G-representation $\mathbb{R}^{n} \otimes \mathfrak{m}$ splits into irreducible components and the corresponding decomposition of $\Gamma$ characterizes the different non-integrable G-structures. We introduce the equivariant maps:

$$
\begin{array}{lll}
\Phi: & \mathbb{R}^{n} \otimes \mathfrak{g} \rightarrow \mathbb{R}^{n} \otimes S^{2}\left(\mathbb{R}^{n}\right), & \Phi(\Sigma)(X, Y, Z):=g(\Sigma(Z)(X), Y)+g(\Sigma(Y)(X), Z), \\
\Psi: & \mathbb{R}^{n} \otimes \mathfrak{m} \rightarrow \mathbb{R}^{n} \otimes S^{2}\left(\mathbb{R}^{n}\right), & \Psi(\Gamma)(X, Y, Z):=g(\Gamma(Y)(X), Z)+g(\Gamma(Z)(X), Y) .
\end{array}
$$

It is well known (see [FI]) that a geometric G-structure admits a connection $\nabla$ with totally skew-symmetric torsion if and only if $\Psi(\Gamma)$ is contained in the image of the homomorphism $\Phi$. There is an equivalent formulation of this condition. Indeed, let us introduce the maps

$$
\Theta_{1}: \Lambda^{3}\left(\mathbb{R}^{n}\right) \rightarrow \mathbb{R}^{n} \otimes \mathfrak{m}, \quad \Theta_{2}: \Lambda^{3}\left(\mathbb{R}^{n}\right) \rightarrow \mathbb{R}^{n} \otimes \mathfrak{g}
$$

given by the formulas

$$
\left.\left.\Theta_{1}(T):=\sum_{i}\left(\sigma_{i}\right\lrcorner T\right) \otimes \sigma_{i}, \quad \Theta_{2}(T):=\sum_{j}\left(\mu_{i}\right\lrcorner T\right) \otimes \mu_{j}
$$

where $\sigma_{i}$ is an orthonormal basis in $\mathfrak{m}$ and $\mu_{j}$ is an orthonormal basis in $\mathfrak{g}$. Observe that the kernel of the map $(\Psi \oplus \Phi): \mathbb{R}^{n} \otimes \mathfrak{s o}(n) \rightarrow \mathbb{R}^{n} \otimes S^{2}\left(\mathbb{R}^{n}\right)$ coincides with the image of the map $\left(\Theta_{1} \oplus \Theta_{2}\right): \Lambda^{3}\left(\mathbb{R}^{n}\right) \rightarrow \mathbb{R}^{n} \otimes \mathfrak{s o}(n)$. Consequently, for any element $\Gamma \in \mathbb{R}^{n} \otimes \mathfrak{m}$, the condition $\Psi(\Gamma) \in \operatorname{Image}(\Phi)$ is equivalent to $\Gamma \in \operatorname{Image}\left(\Theta_{1}\right)$.

Theorem 5.1. A G-structure $\mathcal{R} \subset \mathcal{F}\left(M^{n}\right)$ of a Riemannian manifold admits a connection $\nabla$ with totally skew-symmetric torsion if and only if the 1-form $\Gamma$ belongs to the image of $\Theta_{1}$, $\Gamma=\Theta_{1}(T)$. In this case the 3 -form $(-2 \cdot T)$ is the torsion form of the connection.

Consequently, only such geometric types (i. e. irreducible components of $\mathbb{R}^{n} \otimes \mathfrak{m}$ ) are admissible which occur in the G-decomposition of $\Lambda^{3}\left(\mathbb{R}^{n}\right)$. This explains the different behavior of Gstructures with respect to conformal transformations.

Example 5.1. In case of $\mathrm{G}=\operatorname{Spin}(9)$ we have

$$
\mathbb{R}^{16} \otimes \mathfrak{m}=\mathbb{R}^{16} \oplus \Lambda^{3}\left(\mathbb{R}^{16}\right) \oplus \mathcal{P}_{3}\left(\mathbb{R}^{9}\right)
$$


and the $\mathbb{R}^{16}$-component is not contained in $\Lambda^{3}\left(\mathbb{R}^{16}\right)$, i.e., a conformal change of a $\operatorname{Spin}(9)$ structure does not preserve the property that the structure admits a connection with totally skew-symmetric torsion.

Example 5.2. In case of a 7-dimensional $\mathrm{G}_{2}$-structure the situation is different. Indeed, we decompose the $\mathrm{G}_{2}$-representation (see $\mathrm{FI1}$ )

$$
\Lambda^{3}\left(\mathbb{R}^{7}\right)=\mathbb{R}^{1} \oplus \mathbb{R}^{7} \oplus \Lambda_{27}^{3}, \quad \mathbb{R}^{7} \otimes \mathfrak{m}=\mathbb{R}^{1} \oplus \mathbb{R}^{7} \oplus \Lambda_{14}^{2} \oplus \Lambda_{27}^{3}
$$

and, consequently, a conformal change of a $\mathrm{G}_{2}$-structure preserves the property that the structure admits a connection with totally skew-symmetric torsion.

Example 5.3. Let us consider Spin(7)-structures on 8-dimensional Riemannian manifolds. The subgroup $\operatorname{Spin}(7) \subset \mathrm{SO}(8)$ is the real $\operatorname{Spin}(7)$-representation $\Delta_{7}=\mathbb{R}^{8}$. The complement $\mathfrak{m}=\mathbb{R}^{7}$ is the standard 7-dimensional representation and the $\operatorname{Spin}(7)$-structures on an 8-dimensional Riemannian manifold $M^{8}$ correspond to the irreducible components of the tensor product

$$
\mathbb{R}^{8} \otimes \mathfrak{m}=\mathbb{R}^{8} \otimes \mathbb{R}^{7}=\Delta_{7} \otimes \mathbb{R}^{7}=\Delta_{7} \oplus \mathrm{K},
$$

where $\mathrm{K}$ denotes the kernel of the Clifford multiplication $\Delta_{7} \otimes \mathbb{R}^{7} \rightarrow \Delta_{7}$. It is well known that $\mathrm{K}$ is an irreducible Spin-representation. Therefore, there are only two basic types of Spin(7)structures (see $[$ Fer $]$ ). On the other hand, the map $\Lambda^{3}\left(\mathbb{R}^{8}\right) \rightarrow \mathbb{R}^{8} \otimes \mathfrak{m}$ is injective and the $\operatorname{Spin}(7)$-representation $\Lambda^{3}\left(\mathbb{R}^{8}\right)=\Lambda^{3}\left(\Delta_{7}\right)$ splits again into the irreducible components

$$
\Lambda^{3}\left(\Delta_{7}\right)=\Delta_{7} \oplus \mathrm{K},
$$

i. e., $\Lambda^{3}\left(\mathbb{R}^{8}\right) \rightarrow \mathbb{R}^{8} \otimes \mathfrak{m}$ is an isomorphism. Theorem 5.1 yields immediately that any $\operatorname{Spin}(7)$ structure on an 8-dimensional Riemannian manifold admits a connection with totally skewsymmetric torsion (see [ $\mathrm{IV}]$ ). We remark that $n=8$ is the smallest dimension where this effect can occur. Indeed, let $\mathrm{G} \subset \mathrm{SO}(n)$ be a subgroup of dimension $\mathrm{g}$ and suppose that any G-structure admits a connection with totally skew-symmetric torsion, i.e., the map $\Lambda^{3}\left(\mathbb{R}^{n}\right) \rightarrow$ $\mathbb{R}^{n} \otimes \mathfrak{m}$ is surjective. On the other side, the isotropy representation $\mathrm{G} \rightarrow \mathrm{SO}(\mathfrak{m})$ of the compact Riemannian manifold $\mathrm{SO}(n) / \mathrm{G}$ is injective. Consequently, we obtain the inequalities

$$
\frac{1}{3}\left(n^{2}-1\right) \leq \mathrm{g} \leq \frac{1}{2}\left(n^{2}-3 n+2\right) .
$$

The minimal pair satisfying this condition is $n=8, \mathrm{~g}=21$. Using not only the dimension of the G-representation one can exclude other dimensions, for example $n=9$.

\section{REFERENCES}

[Agri] I. Agricola, Connections on naturally reductive spaces, their Dirac operator and homogeneous models in string theory, to appear.

[DoFi] I.G. Dotti and A. Fino, Hyperkähler torsion structures invariant by nilpotent Lie groups, math.dg/0112166.

[Fer] M. Fernandez, A classification of Riemannian manifolds with structure group Spin(7), Ann. de Mat. pura ed applicata vol. CXLIII (1986), 101-122.

[Fri1] Th. Friedrich, Weak Spin(9)-structures on 16-dimensional Riemannian manifolds, Asian Journ. Math. 5 (2001), 129-160.

[FI1] Th. Friedrich and S. Ivanov, Parallel spinors and connections with skew-symmetric torsion in string theory, math.dg/0102142.

[FI2] Th. Friedrich and S. Ivanov, Almost contact manifolds and type II string equations, math.dg/0111131.

[FI3] Th. Friedrich and S. Ivanov, Killing spinor equations in dimension 7 and geometry of integrable $G_{2}$ manifolds, math.dg/0112201.

[FH] W. Fulton and J. Harris, Representation theory, Graduate texts in mathematics, Springer-Verlag New York, 1991.

[Gray] A. Gray, Weak holonomy groups, Math. Z. 123 (1971), 290-300.

[HP] P. S. Howe and G. Papadopoulos, Finiteness and anomalies in $(4,0)$ supersymmetric sigma models, Nucl. Phys. B 381 (1992), 360.

[Iv] S. Ivanov, Connection with torsion, parallel spinors and geometry of Spin(7)-manifolds, math.dg/0111216. 
[IP] S. Ivanov and G. Papadopoulos, Vanishing theorems and string background, Class. Quant. Grav. 18 (2001), 1089-1110.

[IM] S. Ivanov and I. Minchev, Quaternionic Kähler and hyperKähler manifolds with torsion and twistor spaces, math.dg/0112157.

[SSTP] P. Spindel, A. Sevrin, W. Troost, A. van Proeyen, Extended supersymmetric $\sigma$-models on group manifolds, Nucl. Phys. B 308 (1988), 662-698.

[Stro] A. Strominger, Superstrings with torsion, Nucl. Phys. B 274 (1986), 253-284.

[Ver] M. Verbitsky, Hyperkähler manifolds with torsion, supersymmetry and Hodge theory, math.ag/0112215.

THOMAS FRIEDRICH

Institut Für Reine Mathematik

Humboldt-Universität ZU BerLin

Sitz: WBC AdLERShOF

D-10099 Berlin, Germany

friedric@mathematik.hu-berlin.de 